\title{
ASSESSING OPENSTREETMAP URBAN NETWORK OF ORAN CITY
}

\author{
B. Meguenni ${ }^{1, *}$, M. A. Hafid ${ }^{1}$ \\ ${ }^{1}$ CTS, Centre des Techniques Spatiales, 31002 Arzew Oran, Algeria - (bmeguenni, ahafid)@cts.asal.dz
}

Commission VI, WG VI/4

KEY WORDS: Urban Network, OpenStreetMap, Spatial Data Quality

\begin{abstract}
:
OpenStreetMap (OSM) uses the Open Database License, it is a collaborative project that collects a rich set of vector data provided by volunteers. It is a global collection of mapping data that can be used for a wide variety of purposes. Many third-party online maps are based on OpenStreetMap data. Currently, more and more large organizations are choosing OSM for their maps.

In addition, the analysis of the spatial quality of the OSM data shows that particular care must be taken. However, there are several methods for assessing the quality of the OSM data by comparing the OSM to an authoritative dataset. In this context, it is essential to develop an automatic procedure to improve its spatial quality.

This work proposes a quantitative method for comparing the quality of the OSM and an authoritative data set on urban networks in the city of Oran (Algeria). The procedure is based on python modules in a GIS environment and provides measurements of the spatial accuracy and completeness of the OSM road network. The method is applied to assess the quality of the Oran OSM road network data set through a comparison with the official Algerian dataset. The results show that the OSM's Algerian road network is very complete, but with low spatial accuracy.
\end{abstract}

\section{INTRODUCTION}

\subsection{General background}

The frequency of natural disasters around the world has increased steadily in recent decades. These disasters are damaging social and economic infrastructure in all countries, but their long-term consequences are particularly severe for developing countries. Indeed, disasters have a major impact on development: in some cases, a disaster can erase years of effort, sometimes causing people to regress to lower levels of poverty. Vulnerable populations are, by definition, both the most vulnerable to hazards and the most deprived of means of recovery. Already destitute, they are hit harder than the wealthier populations. Hence the importance to be given to prevention issues.

The vulnerability of populations is also aggravated by a lack of management capacities of the various actors. Indeed, the means of responding to the emergency, the speed of reconstruction, the speed of recovery are different according to the level of development of the countries, which can aggravate the scale of the disaster. To reduce the impact of disasters on vulnerable communities, this work therefore aims to act on both axes: improving disaster prevention and response through a better knowledge of risks and behaviours to be maintained. The objective is to map all data on buildings, roads and major critical infrastructure. The data used to implement the spatial database comes from the OpenStreetMap (OSM) platform. VGI data such as OSM require special geometric and attribute processing to make them reliable for spatial analysis. The data quality indicators identified by TC211(Husen 2018) for the assessment of spatial data are completeness, logical or topological consistency, positional accuracy, semantic accuracy, attribute accuracy, temporal accuracy and lineage, which have been used in various studies A methodology is applied to geometrically correct the OSM data, based on the variable radius buffering technique.

\subsection{Volunteered Geographic Information (VGI)}

The availability of high-resolution satellite images has enabled a large participation of people around the world who are not necessarily GIS specialists to produce spatial data that is increasingly being used in several sectors under open source license. This type of information was termed 'Volunteered Geographical Information' (VGI) by Goodchild, 2007. One of the most well-known VGI projects is the OpenStreetMap (OSM) that provides worldwide free geospatial data representing a variety of features. The OSM data are available under an Open Data Commons Open Database license (ODbL) for reuse, which allows for sharing, creation and adapting of the data unless reuse is attributed (Brassel 1995).

\subsection{OpenStreetMap}

OpenStreetMap is a leading VGI (Volunteered Geographic Information) project, aiming at creating a free editable map of the world. OpenStreetMap (OSM) is an international project founded in 2004 to create a free map of the world, with the main theme of the road network. Data collection is carried out by volunteers from all over the world on roads, railways, rivers, forests, buildings, etc. It is an initiative to create and provide free geographical data, such as transport and communications networks.

OSM data can be integrated into a Geographic Information System (GIS) after processing and formatting to enrich the geometric and semantic information of an existing geographic database. A critical issue for all VGI initiatives is the quality of the information offered. An assessment of the spatial quality of OSM data is therefore essential to integrate it into our GIS dedicated to natural disaster management

\footnotetext{
* Corresponding author
} 


\section{METHODOLOGY}

The following methodology aims to assess the quality elements of OSM road network (tested dataset) against the BDR dataset (reference dataset). To carry out this work, we used Alsat2 satellite panchromatic images of Great Oran taken in 2015. The image have a $2.5 \mathrm{~m}$ resolution.

\subsection{Data collection}

This work applied extrinsic OSM assessment, in which OSM and reference data can be compared to one another.

The objective of this work is to evaluate the spatial coherence of OSM data and more precisely the geometric coherence on the one hand, and to show the potential of OSM maps to contribute to the updating of the existing road database.

The evaluation of geometric consistency requires the comparison of OSM data by matching with another data source in the same area. In our case we will use the large-scale road database as well as the transport database (Transport Directorate) of the city of Oran. This is justified by the fact that free data in general, such as OSM data, give no guarantee in terms of geometric or semantic accuracy, unlike large-scale road comics, or transport comics, where geometric accuracy is directly related to the map scale.

The large-scale Road database is structured as a main map with planimetrically depicted elements, but a relief map represented by contour lines is also available.

The BD-Transport is made up of several layers of information, namely, the road network, transport lines, bus stops, etc. Our work only took into consideration the city's road network.

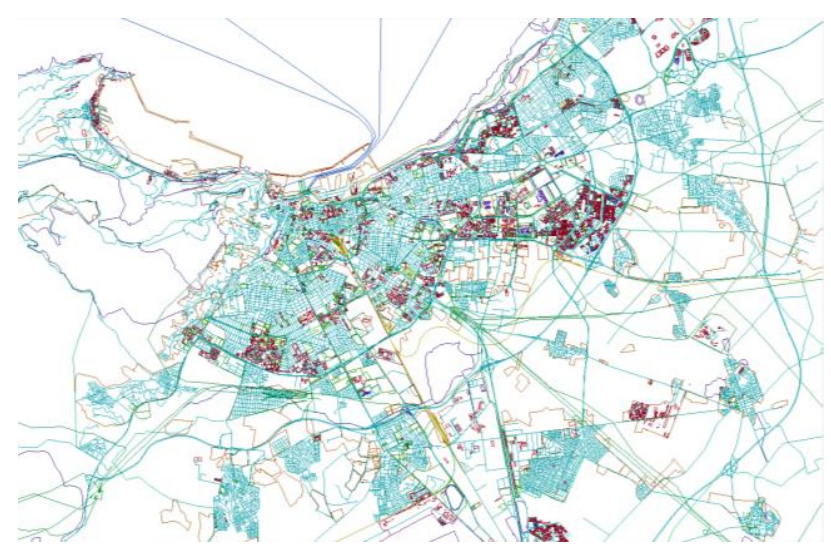

Figure 1. OSM and reference data

\subsection{Study area and data preparation}

The study area used in this study is the city of Oran (Algeria) and its immediate peripheral areas; Oran is the second city in Algeria.

The OSM data sets of Oran were converted into Geodatabase format and visualised in ArcGIS software.

The reference vector data were obtained from the local authority that administers the Oran city, namely "Base de données routière" (BDR).

For comparison, both data were projected onto the Universal transverse Mercator (UTM) World geodetic system WGS84 UTM 30.

Before conducting the quality assessment, the OSM data were pre-processed as below
1- Filtering: the attributes of road and building features were extracted from the OSM datasets.

2- Feature geometric/matching: corresponding roads between the reference vector data and OSM data were identified based on the plugin RoadMatcher for the GIS OpenJump, RoadMatcher is a plugin for the GIS OpenJUMP that allows for automated conflation of geographical topologies as shown in Figure 2.

3- Feature semantic/matching: Corresponding roads roads between reference and OSM datasets were identified based on the semantics keywords as shown in Table 1.

\begin{tabular}{|l|c|c|}
\hline Reference data & OSM data & Matched keywords \\
\hline Primary & Motorway/Primary & Primary Road \\
\hline Secondary & Secondary/ Tertiary & Secondary Road \\
\hline Residential & Residential & Residential Road \\
\hline
\end{tabular}

Table 1. Semantic matching between OSM and reference data

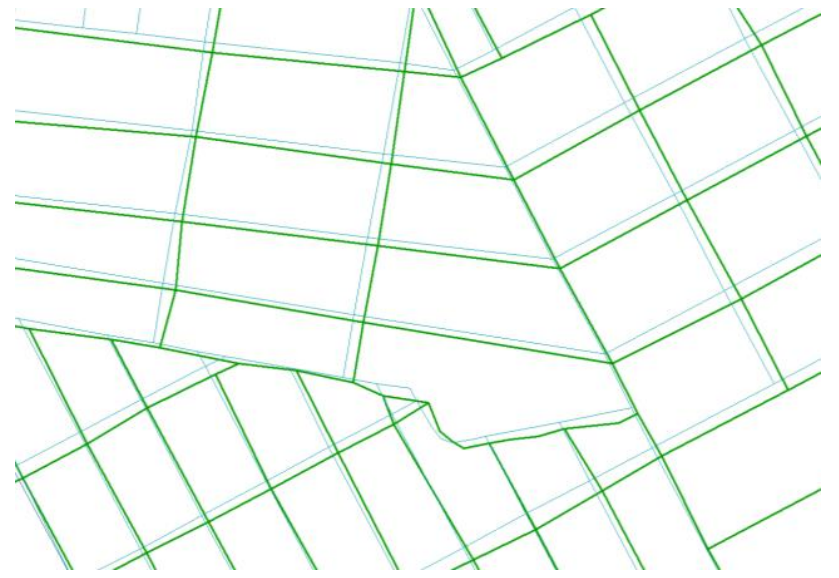

Figure 2. Geometric matching between OSM and reference data

\subsection{Calculating the positional accuracy}

Positional accuracy is the assessment of position of features where relative positional accuracy is the closeness of the relative positions of features to their respective relative positions. The buffer-based method was used to compute the positional accuracy of road features in this work. By using this method, the accuracy is determined by the percentage of OSM road that is within the buffer of the corresponding BDR road feature.

The methodology applied is illustrated in the following organigram, as shown in Figure 3.

First, we create a buffer with an initial radius, and then we establish the matching.

Once the matching is determined by selection by location, with the total inclusion criterion, the relative road sections will be exported to make a joint with their homologues in the BDR Reference Roads table. The corresponding OSM data will then be loaded into a temporary table.

If the overlap is reached (according to the operator's appreciation) the OSM data will be validated and integrated, otherwise, return to step 1 by increasing the search radius with a step, in our case the step is 0.5 


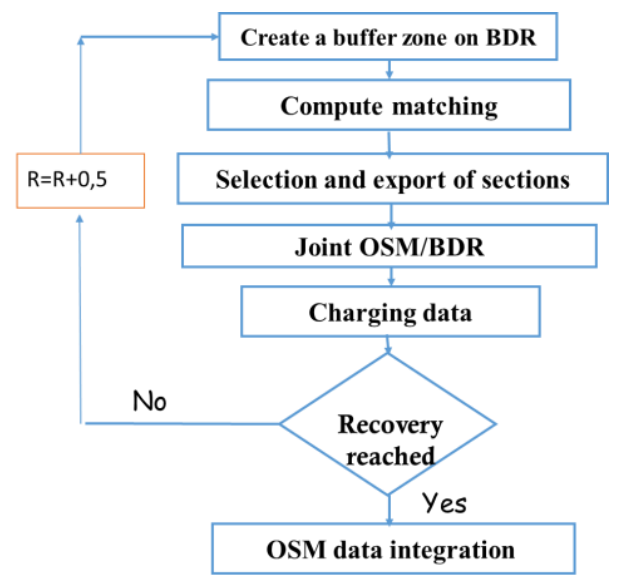

Figure 3. Organigram - calculating matching features

\subsection{Calculating the completeness of features}

The completeness of map data is the most important data quality element next to positional accuracy. Completeness of spatial data describes the existence of features (Kounadi 2009) as compared to ground reality.

For assessing the completeness, an established procedure is used to compare test data with reference data. Features of both datasets were overlaid and the percentage of were calculated using the equation (1) below;

Completeness $\%=((\mathrm{OSM}$ number of section/BDR number of section)*100).

\section{RESULTS AND DISCUSSIONS}

\subsection{Positional accuracy of roads}

To simplify the analysis of the results, we have created the OSM_BDR table, which is used to load the results of each iteration with the radius, which is the matching distance.

The analysis is based on the calculation of the number of entities covered for each radius (distance); the following table illustrates the results.

\begin{tabular}{|r|r|}
\hline Buffer_radius & Number of OSM segments covered \\
\hline 0,5 & 50 \\
\hline 1 & 210 \\
\hline 1,5 & 459 \\
\hline 2 & 755 \\
\hline 2,5 & 1095 \\
\hline 3 & 1489 \\
\hline 3,5 & 1838 \\
\hline 4 & 2184 \\
\hline 4,5 & 2509 \\
\hline 5 & 2807 \\
\hline 5,5 & 3028 \\
\hline 6 & 3251 \\
\hline 6.5 & 3413 \\
\hline 7 & 3547 \\
\hline 7.5 & 3643 \\
\hline 8 & 3733 \\
\hline
\end{tabular}

Table 2. Matching calculation based on the number of OSM sections covered
In this study, the correctness of road class was calculated by comparing the length of road with the correct 'type road' against BDR dataset using equation (2) below;

Correctness $\%=((\mathrm{A} / \mathrm{B}) * 100)$

Where,

$\mathrm{A}=$ OSM road length with correct class

$\mathrm{B}=\mathrm{OSM}$ total road length

The figure below illustrates the length percentage of the OSM dataset included in buffers of different widths around the REF dataset.

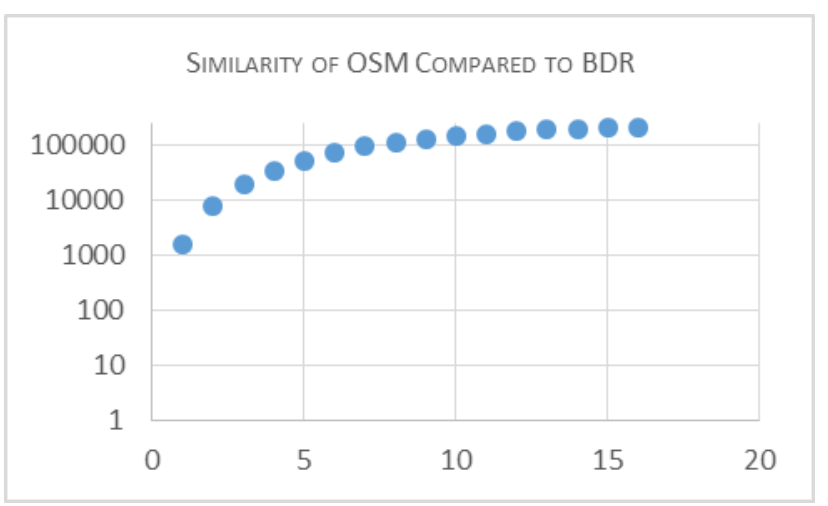

Figure 4. Similarity of OSM compared to BDR.

\section{CONCLUSION}

Despite all the work done in recent years, the updating of databases with OSM data remains a complex and not perfectly solved problem.

In this work, we first focused on updating the Road Database using OSM data. This database will be shared online to assist in traffic management in the event of a natural disaster.

We proposed a method for evaluating OSM data to update the large-scale road database. The results are quite acceptable for a first test.

\section{REFERENCES}

Ramaprasad, Priya, 2019. Volunteered Geographic Information System and Its Contribution in Service Sector Employment, IntechOpen, DOI: 10.5772/intechopen.82004. Available from: https://www.intechopen.com/online-first/volunteeredgeographic-information-system-and-its-contribution-in-servicesector-employment (January 9th 2019)

ODC Open Database License (ODbL), Version 1.0. Available online:https://opendatacommons.org/licenses/odbl/ (accessed on 30 March 2017).

Goodchild, M. F. 2007. Citizens as sensors: the world of volunteered geography. GeoJournal, 69(4), 211-221. doi:10.1007/s10708-007-9111-y

Brassel, K.; Bucher, F.; Stephan, E.M.; Vckovski, A. 1995 Completeness. In Elements of Spatial Data Quality; Guptill, S.C., Morrison, J.L., Eds.; Elsevier Science Ltd.: Exeter, UK,; Chapter five, pp. 81-108.

Kounadi, O. 2009, Assessing the quality of OpenStreetMap data geographical information science, MSc Geographical Information Science, University College of London. 
Husen, S. 2018, The Quality of OpenStreetMap in Malaysia: A Preliminary Assessment, https://doi.org/10.5194/isprs-archivesXLII-4-W9-291-2018, The International Archives of the Photogrammetry, Remote Sensing and Spatial Information Sciences, Volume XLII-4/W9. 